\title{
Ultradrawing of High Molecular Weight Polyethylene Films Produced by Gelation/Crystallization from Solution: Effect of the Number of Entanglements
}

\author{
Masaru MAtsuo, ${ }^{*}$ Chie SAwATARI, Masayasu IIDA, ${ }^{* *}$ \\ and Morihiro YONEDA \\ Department of Clothing Science, Faculty of Home Economics, \\ Nara Women's University, Nara 630, Japan
}

(Received February 26, 1985)

\begin{abstract}
This paper describes the morphological properties of gel films of ultrahigh molecular weight $\left(6 \times 10^{6}\right)$ polyethylene by small angle X-ray scattering (SAXS), wide angle X-ray diffraction (WAXD), and scanning electron microscopy. The gel films were prepared by crystallization from solution with critical concentration associated with the maximum drawability of the dry gel films. Through a series of experimental results, it turns out that the number of entanglements causes significant changes in the profile of SAXS intensity distribution and in the orientation of crystallites estimated by WAXD patterns. Furthermore, stress-strain curves were dependent upon an increase of the entanglements. Based on the above results, it may be concluded that a suitable level of entanglements between crystal lamellae plays an important role for transmitting drawing force as intermolecular crosslinks in an ultradrawing process.
\end{abstract}

KEY WORDS Ultra High Molecular Polyethylene / Dry Gel Films /

Critical Concentration / Entanglements / Intermolecular Crosslinks /

Ultradrawing of high molecular weight polyethylene was first carried out by Smith and Lemstra $^{1-3}$ through gelation/crystallization from dilute solution on the assumption that the maximum achievable draw ratio depends principally on the concentration of the solution from which the gel was made. This concept was attributed to a reduced-number of entanglements per molecule in solution cast/spun polymers in comparison with those obtained from the melt. Actually, Smith et al. ${ }^{3}$ succeeded in producing fibers with a draw ratio of 130 and the consequent Young's modulus of $150 \mathrm{GPa}$.

Based on the above concept, Matsuo et al. ${ }^{4}$ proposed one approach to determine optimum entanglements which can be realized by a proper choice of the solution concentration through measurement of solution viscosity for the purpose of preparing gel films with high drawability. The experimental results, proved that there exists an apparent critical concentration associated with an empirical correlation between the mechanical properties of the optimum gel and the characteristics of the dilute polymer solution. The plot of the reduced viscosity $\eta_{\mathrm{sp}} / c$ against the concentration $c$ could be classified into two reduced viscosity regimes, one of weak concentration dependence and the other of strong concentration dependence. The intersection between two concentration regimes was found to correspond to the concentration with the maximum drawability of the dry gel films experimentally. Accordingly, the intersection of the concentration was defined as the critical concen-

* To whom correspondence should be addressed.

** Department of Chemistry, Faculty of Science, Nara Women's University, Nara 630, Japan. 
tration to prepare dry gel films with high drawability. According to our previous experimental results ${ }^{4}$ for polyethylene with a high molecular weight of $6 \times 10^{6}$, the critical concentration was almost $0.4 \mathrm{~g} / 100 \mathrm{ml}$. The dry gel films prepared from a solution at the critical concentration $(0.4 \mathrm{~g} / 100 \mathrm{ml})$ could be readily elongated up to a draw ratio of $\lambda=300$ at $135^{\circ} \mathrm{C}$. For such ultradrawn films, the Young's modulus and tensile strength attained maximum values of 202 and $6.2 \mathrm{GPa}$, respectively. These are among the highest values ever reported. Accordingly, it is thought that this critical concentration is appropriate to ensure the existence of a suitable level of the entanglements that act as intermolecular crosslinks and effectively transmit the drawing force of dry gel films.

The importance of a suitable level of entanglements was discussed in terms of morphological aspect using wide angle $\mathrm{X}$-ray diffraction (WAXD) and small angle X-ray scattering (SAXS) patterns. $^{5-7}$ The two X-ray patterns indicated that the dried gel film was composed of crystal lamellae that were highly oriented with their large flat faces parallel to the film surface, and that the crystal $c$-axes were oriented perpendicular to the large flat faces within the lamellar crystals. The gel films are therefore similar to mats of single crystals. Judging from the two X-ray patterns, it is noted that as the lamellae with a folded structure are not interconnected by the chain molecules in the lower concentration regime, chain slippage must occur in the transformation process to a fibrous texture, while as small lamellar crystals crystallized from the higher concentration regime are interconnected strongly by the chain molecules, the high entanglement density hampers the achievement of high draw ratio. This indicates that the critical concentration is probably expected to be somewhere between these two regimes. However, there have been no reports about the dependence of the morphological properties of crystal lamellae on the concentration of so- lution to prepare gel films. Accordingly, it is important to clarify that the crystal lamellae prepared from a solution with the critical concentration $c^{*}$ show the intermediate morphological properties between characteristics of crystal lamellae associated with the concentration above $c^{*}$ and those associated with that below $c^{*}$.

This paper persues the above conceptual correlation on the basis of SAXS intensity distribution characterizing the morphological properties of crystal lamellae. The actual experiments were carried out from two viewpoints: the dependence of the lamellar morphology on the concentration and the dependence of mechanical properties on an increase in entanglements due to the annealing effect of the original dry gel films. The dry gel films were elongated at room temperature to prevent an increase in entanglements arising in the preheated process prior to drawing for the purpose of discussing mechanical properties, crystal orientation, and drawability with respect to the number of entanglements of original undeformed gel films.

\section{EXPERIMENTAL}

A linear polyethylene with a molecular weight of $6 \times 10^{6}$ (Hercules 1900/90189) was used as a specimen. The solvent was decalin. The solution containing $0.4 \mathrm{~g} / 100 \mathrm{ml}$ of polyethylene and $0.1 \% \mathrm{w} / \mathrm{w}$ of the anti-oxidant di- $t$-butyl- $p$-cresol was prepared by heating the well-blended polymer/solvent mixture at $135^{\circ} \mathrm{C}$ for $40 \mathrm{~min}$ under nitrogen. The above concentration of $0.4 \mathrm{~g} / 100 \mathrm{ml}$ was the critical one obtained by the measurement of solution viscosity. ${ }^{4}$ The homogenized solution was poured into an aluminum tray that was surrounded by ice water to form a gel. The decalin was allowed to evaporate from the gels under ambient condition for 30 days. The nearly dried film, which had a thickness of about $300 \mu \mathrm{m}$, was immersed in an excess of ethanol for 30 days to remove the anti-oxidant and 
subsequently vacuum-dried for 1 day to remove residual trace of the decalin-ethanol mixture. The specimens were annealed in a silicone oil bath in the temperature range from 20 to $130^{\circ} \mathrm{C}$ and subsequently quenched at room temperature. The specimens were elongated at $20^{\circ} \mathrm{C}$ with an Instron tester at the same cross head speed $(5 \mathrm{~mm} / \mathrm{min})$. The initial dimensions of the sample were: length $20 \mathrm{~mm}$; width $4 \mathrm{~mm}$. These conditions were the same as previously. ${ }^{6}$

The meridional SAXS intensity distribution was detected with a position sensitive proportional counter (PSPC) and a $12 \mathrm{~kW}$ rotating anode X-ray generator (Rigaku RAD-rA operated at $200 \mathrm{~mA}$ at $40 \mathrm{kV}$ ). The measurement was carried out by point forcus with a three-slit system. The $\mathrm{Cu} K_{\alpha} \mathrm{X}$-ray source was monochromatized with a plate-like graphite monochrometer. The X-ray exposure times were $4 \times 10^{5} \mathrm{~s}$ for each specimen.

Density of films was measured by a pycnometer with chlorobenzene-toluene as a medium instead of xylene-carbon tetrachloride in the previous work. ${ }^{4}$ When xylene-carbon tetrachloride was used, the density for the drawn specimen above $\lambda=200$ was equal to unity corresponding to the theoretical density of polyethylene crystal within the experimental error. We doubt whether xylene-carbon tetrachloride is suitable as a medium to measure the density of polyethylene, because of preferential absorption by polyethylene of one liquid. According to Blackadder et al. ${ }^{8}$ and Bhateja, ${ }^{9}$ the mixture of chlorobenzene and toluene was used as a good medium to avoid a density differential between the imbibed and the ambient liquids around the specimen. Therefore chlorobenzene-toluene was used as the medium in this experiment to prevent the preferential absorption. Incidentally, great care was taken to remove the anti-oxidant, since the residual anti-oxidant is very dependent on the density. Then the drawn specimens were cut into fragments and they were immersed in an excess of ethanol for 10 days prior to measur- ing the density and subsequently vacuum-dried for 1 day. The crystallinity for specimens above $\lambda=200$ was $97-98 \%$ and that for undrawn specimens was $81 \%$.

\section{RESULTS AND DISCUSSION}

Figure 1 shows SAXS intensity distribution in the meridional direction for the specimens crystallized for solutions with $0.1,0.4$, and $0.6 \mathrm{~g} / 100 \mathrm{ml}$ concentrations, in which $0.4 \mathrm{~g} / 100 \mathrm{ml}$ corresponds to the apparent critical concentration of the solution discussed already. ${ }^{4}$ In this system, an incident beam was directed parallel to the film surface. As can be seen in Figure 1, the scattered intensity for all the specimens shows up to the fourth order maxima whose magnitude decreases with increasing scattering angle but the scattered maxima become indistinct with increasing the concentration of solution. This means that with increasing the concentration, there is an increase in the orientational disorder and/or thickness fluctuations of the crystal lamellae

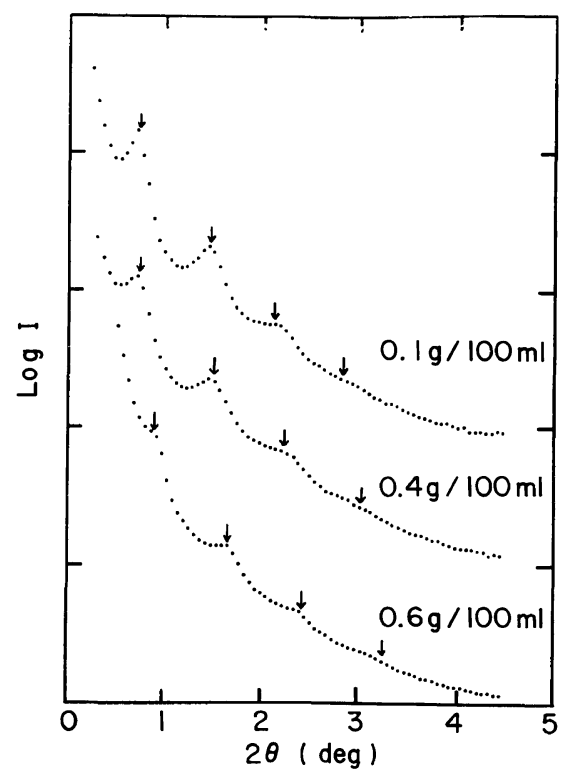

Figure 1. Three SAXS intensity distributions as a function of twice the Bragg angle $(2 \theta)$ in the meridional direction for the specimens crystallized from solutions with $0.1,0.4$, and $0.6 \mathrm{~g} / 100 \mathrm{ml}$ concentrations. 
Table I. Long periods as a function of concentration

\begin{tabular}{cc}
\hline Concentration & Long period \\
$\mathrm{g} / 100 \mathrm{ml}$ & $\AA$ \\
\hline 0.1 & 122 \\
0.4 & 119 \\
0.6 & 106 \\
\hline
\end{tabular}

that were oriented with their flat faces parallel to the film surface. Moreover, the scattering maxima move close to the center of the scattering angle as the concentration decreases. This indicates an increase in the fold period (long spacing), which is listed in Table I.

In the previous work, we proposed one concept for drawability of dry gel films. That is, in the regime of low concentration such as $0.1 \mathrm{~g} / 100 \mathrm{ml}$, most of chain molecules are random coils having coupling entanglements which will be predominantly intramolecular in nature. Therefore, it would be expected that when each chain molecule is completely incorpolated into lamellar crystals, the lamellae are not interconnected by the chain molecules. In such a case, the dry gel films are similar to mats of single crystals. This is in good agreement with the profile of the SAXS intensity distribution whose maxima are the clearest and the long period is the longest among the three SAXS intensity profiles. On the other hand, solutions corresponding to the regime of high concentration such as $0.6 \mathrm{~g} / 100 \mathrm{ml}$ are thought to consist of interpenetrating random coils which form a large number of coupling entanglements that are both intra- and intermolecular. Accordingly, the molecular chains cannot form large lamellae because of the large number of entanglements; the resulting small lamellar crystals are interconnected by the chain molecules. The profile of the SAXS intensity distribution satisfies this concept; the long period is the shortest and each height is least among the three intensity profiles in Figure 1.

At the critical concentration around

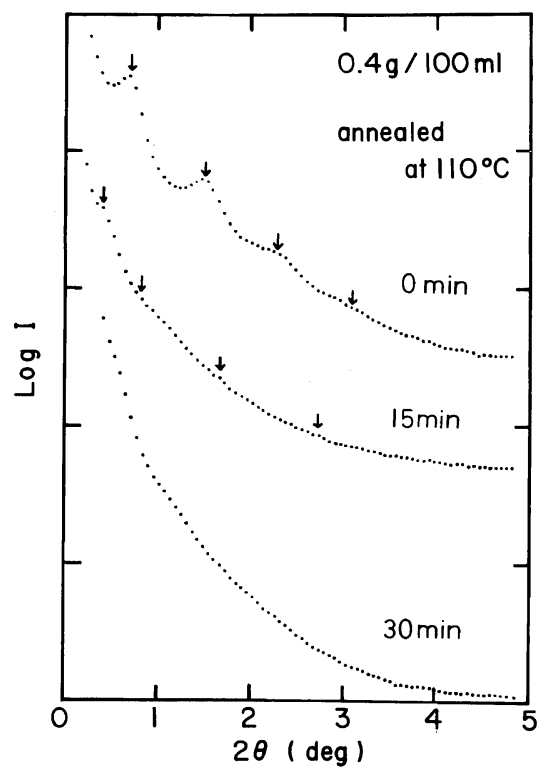

Figure 2. Three SAXS intensity distributions as a function of twice the Bragg angle $(2 \theta)$ in the meridional direction for the specimens annealed at $110^{\circ} \mathrm{C}$ for 0,15 , and $30 \mathrm{~min}$.

$0.4 \mathrm{~g} / 100 \mathrm{ml}$, it would be expected that there exists a suitable level of the entanglements that act as interlamellar crosslinks and effectively transmit the drawing force and then the possibility that polymer chains wiggle through each other without interconnection is very few. This is obviously different from the deformation mode of single crystal mats. Here the crystal lamellae in dry gel films produced from the critical concentration have the intermediate properties between characteristics of crystal lamellae prepared from $0.1 \mathrm{~g} / 100 \mathrm{ml}$ and those prepared from $0.6 \mathrm{~g} / 100 \mathrm{ml}$ solutions. The SAXS profile is in good agreement with the above expectation. Namely, as shown in Figure 1 and Table I, peak height and long period of the specimen concerning $0.4 \mathrm{~g} / 100 \mathrm{ml}$ show each middle value between the results concerning 0.1 and $0.6 \mathrm{~g} / 100 \mathrm{ml}$, which supports the maximum drawability of the films prepared from the critical concentration $(0.4 \mathrm{~g} / 100 \mathrm{ml})$.

Figure 2 shows SAXS intensity distribution 
from dry gel films crystallized from the solution with $0.4 \mathrm{~g} / 100 \mathrm{ml}$ concentration as a function of annealing time. The annealing temperature was $110^{\circ} \mathrm{C}$. This experiment was carried out to obtain more quantitative information in comparison with the photographic experiments in the previous works. ${ }^{5-7}$ As illustrated in Figure 2, the scattering maxima of the intensity distribution become indistinct with increasing the annealing time. On annealing for $30 \mathrm{~min}$, the profile exhibited a monotonically decreasing curve and similar profiles were observed for all the specimens annealed beyond $1 \mathrm{~h}$. The long periods for various times are listed in Table II. The increase in the fold period and orientational disorder and/or thickness fluctuations of crystal lamellae facilitate the drawability. It would be expected that such morphological change due to the annealing plays an important role to increase the number of entanglements and tie molecules. Accordingly, it causes significant increase in stress under elongation with increasing the annealing time, as will be discussed later.

Figure 3 shows the annealing time depen-

Table II. Long periods as a function of annealing time

\begin{tabular}{cc}
\hline Annealing time & $\frac{\text { Long period }}{\min }$ \\
\cline { 2 - 2 } & $\AA$ \\
15 & 119 \\
30 & 220 \\
\hline
\end{tabular}

dence of the nominal stress-strain curves, in which the values of the tensile force were divided by the cross sectional area of the original specimens in determining the stress corresponding to each strain. The drawability was promoted by the annealing time. The probability of successfully drawing up to $\lambda=20$ was highest when the sample annealed for $8 \mathrm{~h}$ was stretched. The stress increased with increasing the annealing time. Such a mechanical property supports the increase in entanglements postulated from the profile of the SAXS intensity distribution in Figure 2. As illustrated in Figure 3, the stress of all specimens decreased slightly with increasing elongation at initial strain and increased again with the elongation. Interestingly, the stress for all the specimens is lower than that measured for the specimens crystallized from the solution with $0.5 \mathrm{~g} / 100 \mathrm{ml}$ concentration discussed in the previous paper $^{6}$ (see Figure 1 in ref 6). This is probably due to the difference of the number of entanglements depending upon the concentration of solution to prepare the gel. Considering the reduced viscosity $\eta_{\mathrm{sp}} / c$ plotted against the concentration $c, 0.5 \mathrm{~g} / 100 \mathrm{ml}$ is apart from the regime which is defined as the critical concentration. The value of $\eta_{\mathrm{sp}} / c$ is much higher than that at $0.4 \mathrm{~g} / 100 \mathrm{ml}$ because of the interpenetrating random coils which form a large number of coupling entanglements.

In order to obtain more conclusive evidence for the effect of entanglements on the mor-

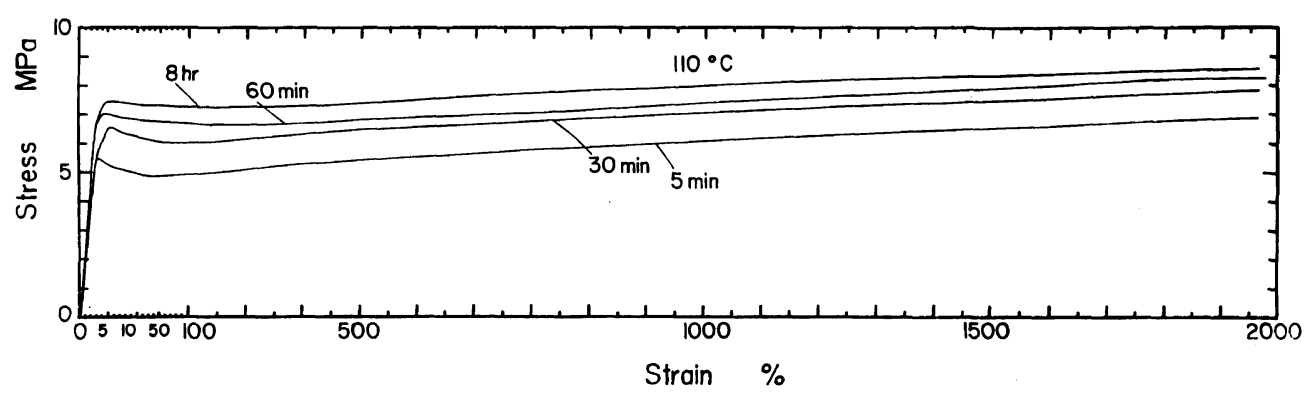

Figure 3. Annealing-time dependence of stress-strain curves recorded at $20^{\circ} \mathrm{C}$ for dry gel films annealed at $110^{\circ} \mathrm{C}$. 


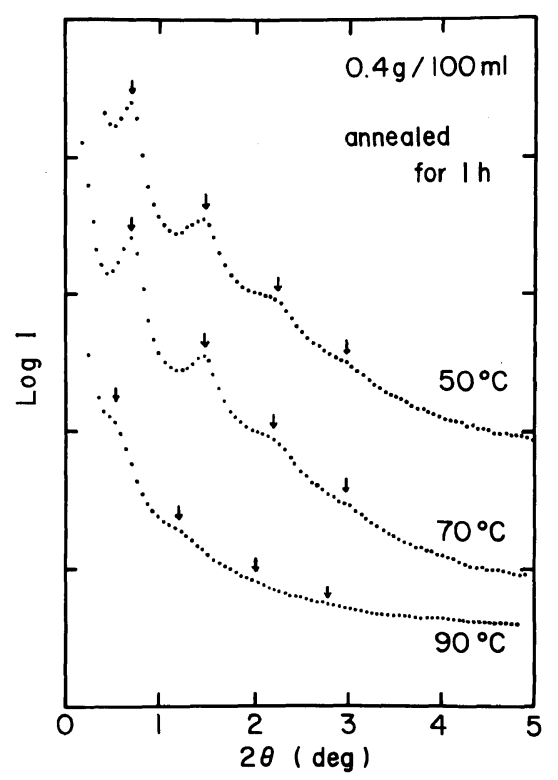

Figure 4. Three SAXS intensity distributions as a function of twice the Bragg angle $(2 \theta)$ in the meridional direction for the specimens annealed at 50, 70, and $90^{\circ} \mathrm{C}$ for $1 \mathrm{~h}$.

phological property of crystal lamellae, the SAXS intensity distribution was measured as a function of the annealing temperature and its correlation is discussed in relation to the mechanical properties. Figure 4 shows a SAXS intensity distribution for the specimens prepared as a function of the annealing temperature. The annealing time was fixed to be $1 \mathrm{~h}$. As illustrated in Figure 4, the profile is hardly affected by the annealing temperature below $70^{\circ} \mathrm{C}$. That is, the two curves at 50 and $70^{\circ} \mathrm{C}$ show similar profiles and the long periods estimated from the scattering maxima are about $120 \AA$. In contrast, the profile at $90^{\circ} \mathrm{C}$ shows indistinct scattering maxima and the long period is $150 \AA$. Incidentally, when the dry gel films were annealed for $1 \mathrm{~h}$ at 110 and $130^{\circ} \mathrm{C}$, the curves monotonically decrease with increasing scattering angle. In this case, it was impossible to estimate the long period.

Figure 5 shows the change in crystallinity with an increase of annealing temperature. The crystallinity tends to increase with increasing

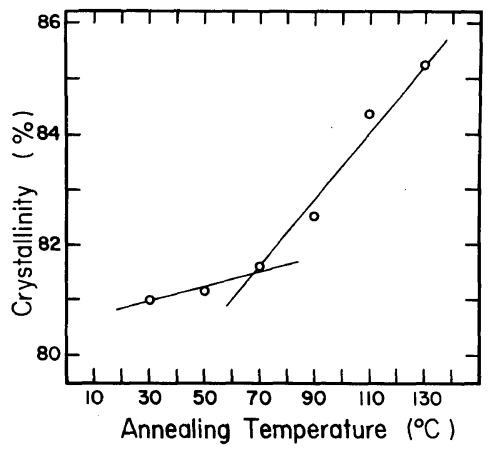

Figure 5. Crystallinity $v s$. annealing temperature for dry gel films.

the annealing temperature and there exists an intersection where there is an abrupt change in crystallinity around $70^{\circ} \mathrm{C}$. Here it should be noted that the considerable change in the profile of the SAXS intensity distribution, associated with the increase in the fold period and orientational disorder and/or thickness fluctuation, was observed at temperatures below and above $70^{\circ} \mathrm{C}$ corresponding to the abrupt change in crystallinity as shown in Figure 5. Therefore, the increase in crystallinity is due to the decrease of fold loops belonging to amorphous regions when molecular chains are reformed as crystal lamellae having a longer folded period than the original ones through the annealing process. In this process, the disorder in size and orientational fluctuation of crystal lamellae become considerable.

Figure 6 shows stress-strain curves for the dry gel films annealed at various temperatures. The stress increases with increasing the temperature and the number of entanglements is associated with the increase in stress. This indicates that the annealing temperature causes significant effect to increase the number of entanglements characterized by the disappearance of the scattering maxima of SAXS intensity distribution. Particularly, the stress of the films annealed at $130^{\circ} \mathrm{C}$ showed considerable large values and the profile of SAXS intensity distribution of original dry gel films 
Ultradrawing of High Molecular Weight PE Films

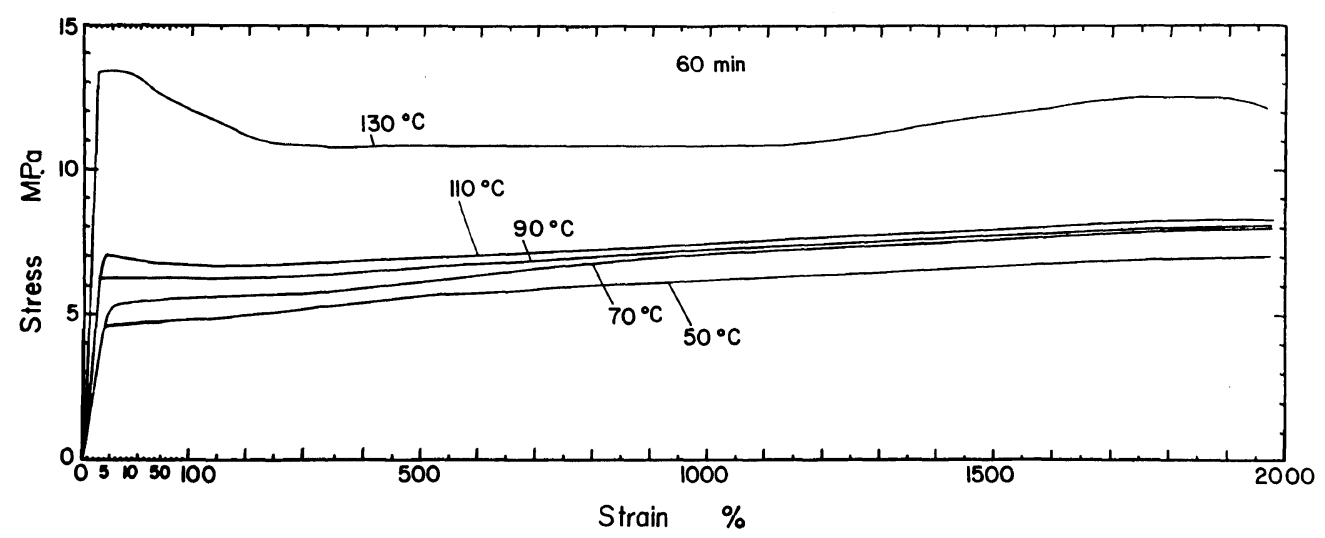

Figure 6. Annealing temperature dependence of stress-strain curves recorded at $20^{\circ} \mathrm{C}$ for dry gel films annealed for $1 \mathrm{~h}$.

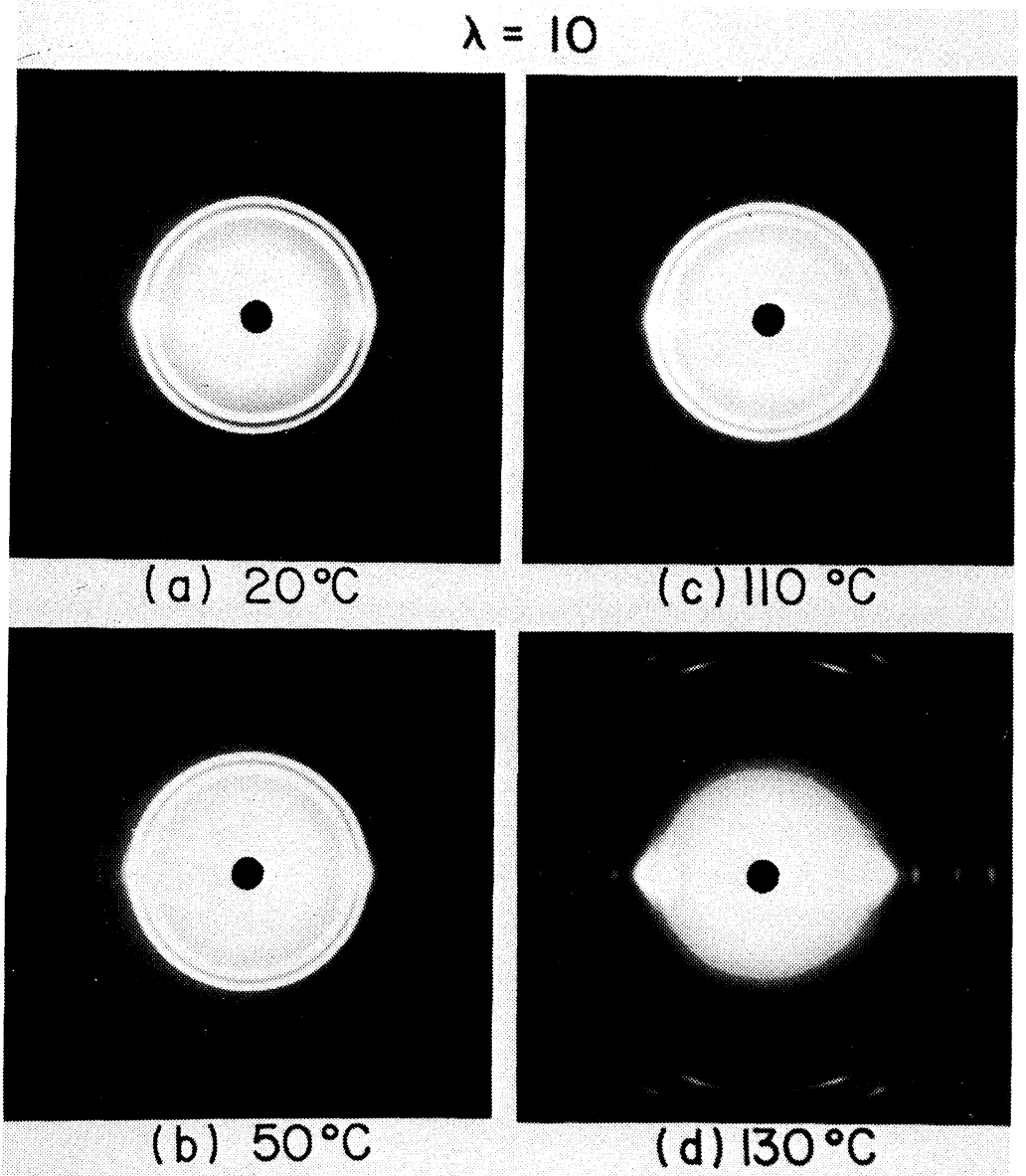

Figure 7. WAXD patterns from specimens drawn to $\lambda=10$ after annealing at temperature below $130^{\circ} \mathrm{C}$. 
annealed showed a further monotonically decreasing curve similar to the one for the films annealed for $30 \mathrm{~min}$ at $110^{\circ} \mathrm{C}$ shown in Figure 2 , although it is not shown in this paper. This is probably due to extreme increase in entanglements and tie molecules in the reformation from original crystal lamellae to ones with longer folded periods, when annealing above $130^{\circ} \mathrm{C}$. If this is the case, it would be expected that the extreme increase in entanglements and tie molecules hamper the crystal transformation from folded to fibrous crystals and therefore the orientational mode of crystallites must be dependent upon the annealing temperature. In order to check this concept, WAXD experiments were carried out for the specimens drawn up to $\lambda=10$ after annealing at different temperatures.

Figure 7 shows WAXD patterns from specimens drawn to $\lambda=10$ after annealing at temperatures below $130^{\circ} \mathrm{C}$ when an incident beam was along the film normal direction (through view). When stretching below $110^{\circ} \mathrm{C}$, the patterns at $\lambda=10$, corresponding to the maximum elongation ratio of melt-crystallized specimens, exhibited strong diffraction rings from the (110) and (200) crystal planes in addition to the equatorial reflection. This indicates the co-existence of two types of orientational modes of crystallites, i.e., random orientation around stretching direction and preferential orientation of the crystal $c$-axes in the stretching direction. This also indicates the coexistence of oriented and unoriented zones within the drawn specimens. In contrast, the WAXD pattern for the specimen annealed at $130^{\circ} \mathrm{C}$ showed strong equatorial reflections and very weak diffraction rings. This indicates that the preferential orientation of the crystal $c$-axes in the stretching direction becomes predominant when the annealing temperature is $130^{\circ} \mathrm{C}$. Based on a number of reports about crystal orientation within polyethylene spherulites, it is concluded that such a preferentially orientational mode of the crystal $c$-axes is due to the rotation of crystallites but not the

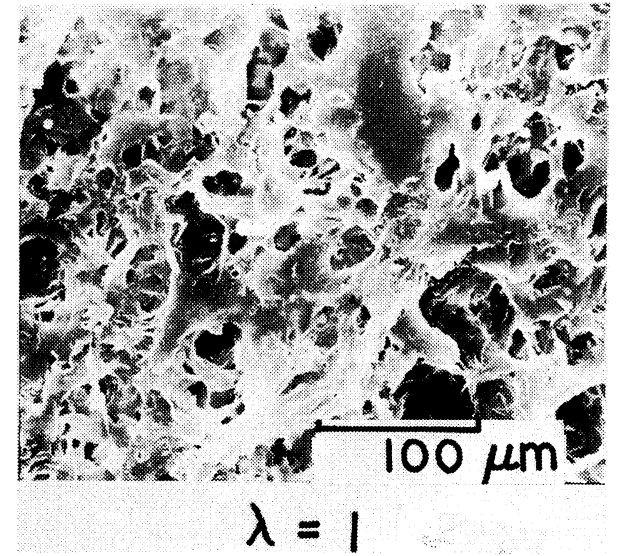

Figure 8. A scanning electron micrograph of an undeformed dry gel film.

crystal transformation from folded to fibrous types. This is based on the fact that when the preferential orientation of the crystal $c$-axes is due to the transformation from folded to fibrous crystals, considerable preferential orientation cannot be realized at such a low elongation ratio as $\lambda=10$. On the other hand, when the preferential orientation of the crystal $c$-axes is due to the rotation of crystallites, high orientation can be realized even at a low elongation ratio, as observed for meltcrystallized specimens. ${ }^{10-12}$

Figure 8 shows scanning electron micrographs of dry gel films prior to stretching and Figure 9 shows the change in the appearance of annealed specimens with stretching at $20^{\circ} \mathrm{C}$. As illustrated in Figure 8, fibrillar texture shows sponge-like tissue. When the annealed specimens were elongated, the deformation behavior of the specimens annealed at 20 and $110^{\circ} \mathrm{C}$ was similar as shown in Figure 9. Namely, the observation revealed that the drawn films consist of two zones unoriented areas with structure corresponding to spongy tissue of the original undrawn specimens and high draw regions oriented parallel to the drawing direction. In highly drawn regions, the width of fibrillar textures decreases with increasing draw ratio and finally the fibrillar textures seem to be disruptively deformed into 


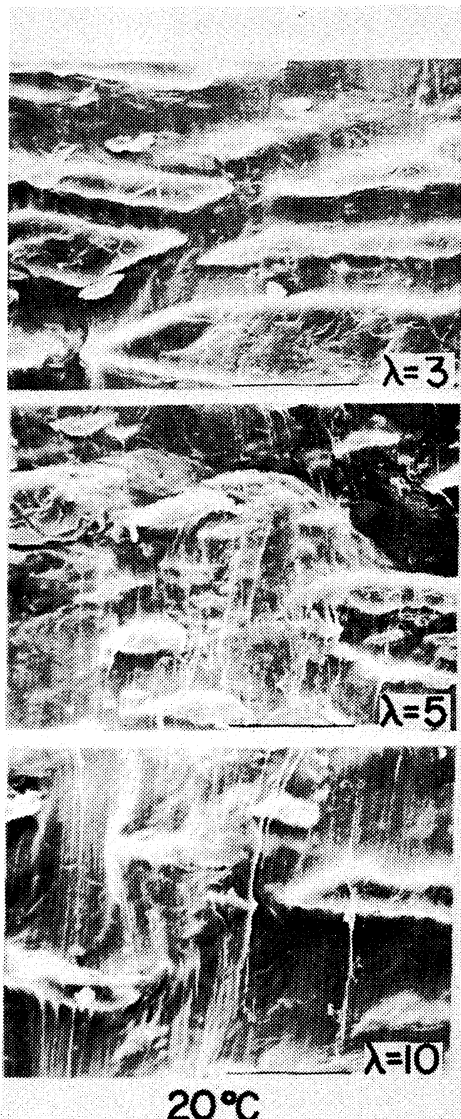

$20^{\circ} \mathrm{C}$

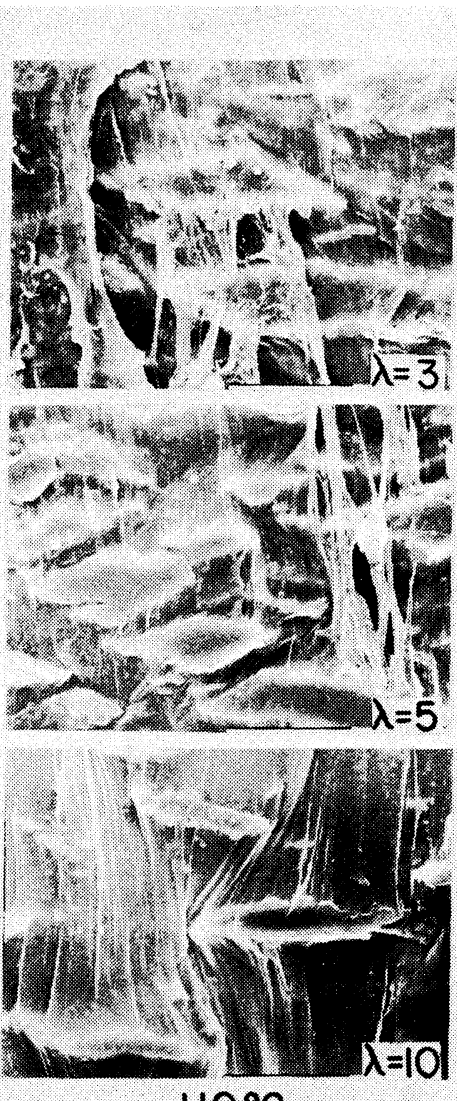

$110^{\circ} \mathrm{C}$

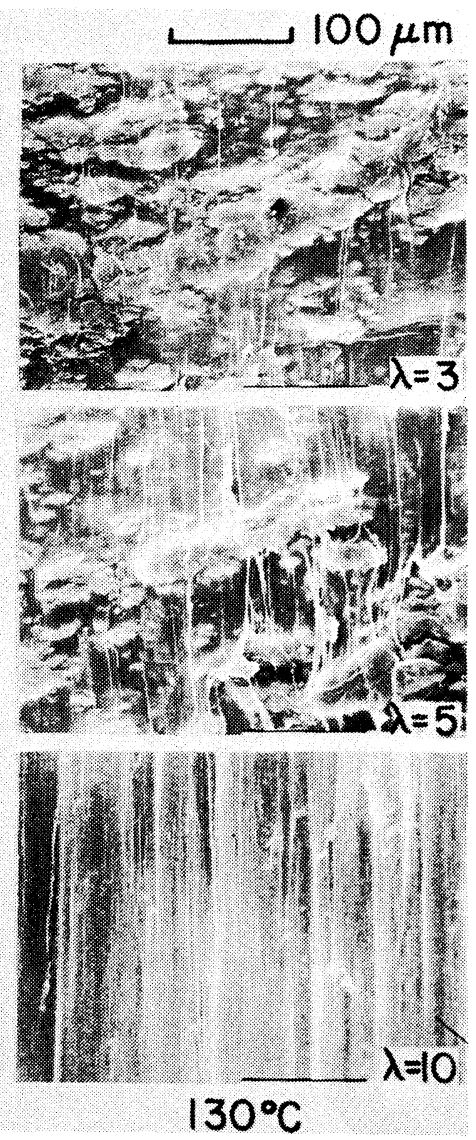

Figure 9. Scanning electron micrographs of dry gel films stretched up to the indicated draw ratio after annealing at 20,110 , and $130^{\circ} \mathrm{C}$.

fine filaments. The filaments are also highly oriented parallel to the drawing direction. As the draw ratio increases, the oriented regions elongate at the expense of the unoriented zones. As for the specimens annealed at $130^{\circ} \mathrm{C}$, the unoriented zone almost disappeared on stretching up to $\lambda=10$. Therefore it may be expected that the crystallites within an unoriented area show random orientation, while those within oriented area show preferential orientation in the stretching direction. Accordingly, in Figure 7, the WAXD patterns exhibit the distinct diffraction rings and spots for the specimens annealed at 20, 50, and $110^{\circ} \mathrm{C}$, while the pattern for the specimen annealed at $130^{\circ} \mathrm{C}$ shows strong spots and very weak diffraction rings.
Figure 10 shows WAXD patterns and the corresponding scanning electron micrographs, in which the pattern and the photograph in the (a) part correspond to the specimen drawn up to $\lambda=15$ at $20^{\circ} \mathrm{C}$ after annealing at $130^{\circ} \mathrm{C}$ for $1 \mathrm{~h}$ and those in the (b) part to the specimen drawn to $\lambda=15$ at $130^{\circ} \mathrm{C}$ after annealing for $15 \mathrm{~min}$. The WAXD patterns exhibited equatorial reflections and diffraction rings. The diffraction rings in the (b) part is more distinct than those in the (a) part and the corresponding photograph shows the existence of unoriented regions surrounded by fine filaments oriented parallel in the stretching direction. As shown in the photograph in the (a) part, unoriented regions almost disappeared and fine filaments are highly oriented parallel to 


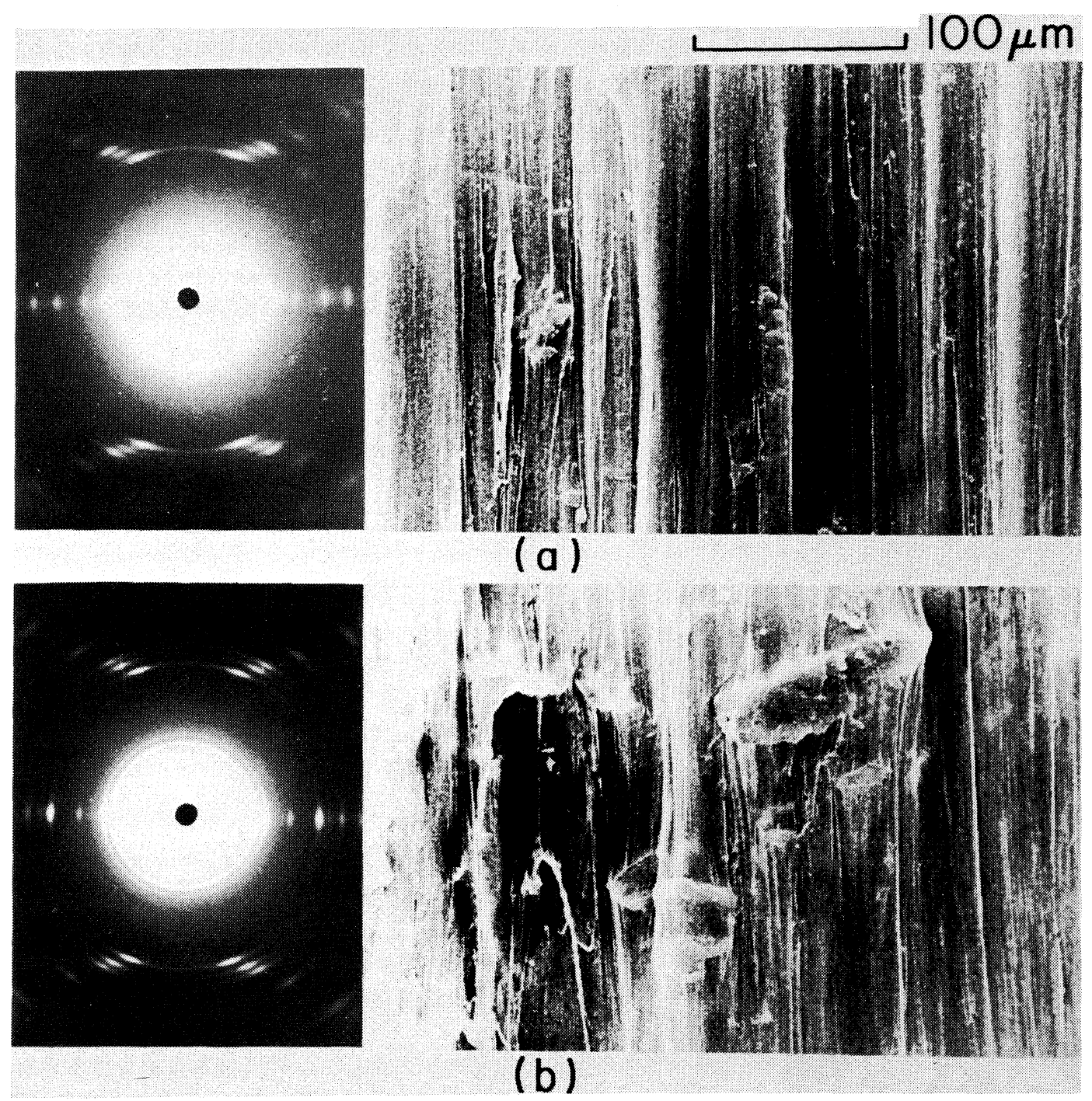

Figure 10. WAXD patterns and the corresponding scanning electron micrographs for the specimens drawn to $\lambda=15$. (a) cold drawing at $20^{\circ} \mathrm{C}$ (b) hot drawing at $130^{\circ} \mathrm{C}$.

the drawing direction. The experimental results in Figure 10 indicate that the degree of crystal orientation for cold drawing is higher than that for hot drawing. However, on cold drawing, the maximum drawabiltiy is only $\lambda=20$. On the other hand, the drawability for hot drawing attained up to $\lambda=300$. Accordingly, it may be expected that the preferential orientation of crystal $c$-axes within fine filaments, on cold drawing, is mainly due to the rotation of the crystallites but not to the transformation from folded to fibrous crystals because entanglements and tie chain molecules are strained and the crystal $c$-axes orient parallel in the drawing direction even at low elongation ratio. In contrast, on hot drawing, the crystallites orient gradually in the drawing direction on the transformation process from folded to fibrous crystals, so that a drawability up to $\lambda=300$ can be realized. 


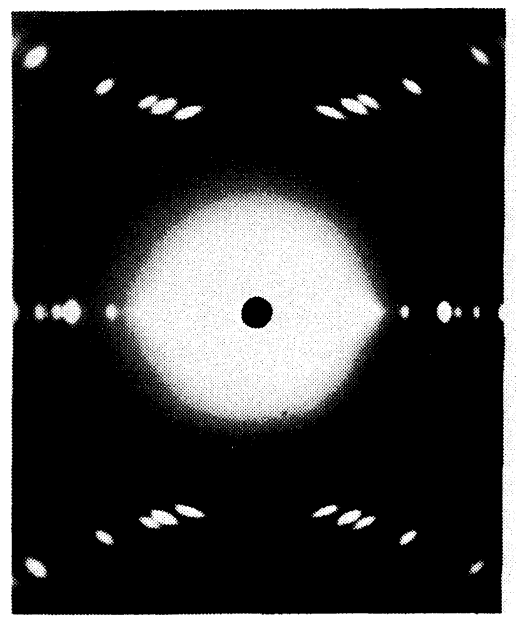

(a) $\lambda=50$

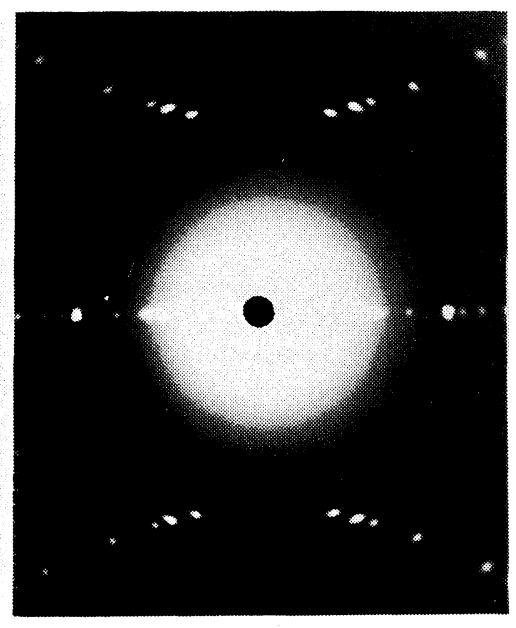

(b) $\lambda=300$

Figure 11. WAXD patterns of dry gel films drawn to (a) $\lambda=50$ and (b) $\lambda=300$ at $135^{\circ} \mathrm{C}$ and piled up four times.

Figure 11 shows WAXD patterns for the specimens drawn up to $\lambda=50$ and 300 at $135^{\circ} \mathrm{C}$. In this case, the elongation was carried out immediately when the temperature in a hot oven became $135^{\circ} \mathrm{C}$. On annealing beyond $15 \mathrm{~min}$, the drawability decreased considerably because of an extreme increase of the number of entanglements. The strong air scattering appeared for a long exposure time $(2 \mathrm{~h})$, since there are very few crystallites within the films with a thickness of $8-10 \mu \mathrm{m}$ (at $\lambda=50$ ) and $3-4 \mu \mathrm{m}$ (at $\lambda=300)$. The air scattering was unavoidable, although four films were piled up to increase the number of crystallites within the irradiated volume of X-ray beam. As illustrated in Figure 11, it is obvious that the crystal $c$-axes are oriented almost perfectly in the drawing direction, because there exist no diffraction rings which appeared in the pattern shown in Figures 9 and 10. Therefore it may be expected that most of the crystals transform from folded to fibrous types and orient in the stretching direction when the specimens were elongated up to $\lambda=50$.

Considering the whole data in this paper, the question is arisen why ultradrawing up to $\lambda=300$ can be achieved at $135^{\circ} \mathrm{C}$ associated with an extreme increase of entanglements. This is due to the fact that drawability is dependent upon the softening of specimens as well as the suitable level of entanglements and $135^{\circ} \mathrm{C}$ is the temperature associated with abrupt softening as indicated from DSC heating curves. ${ }^{13}$ Actually, the achievement of high draw ratio could be realized under the condition that elongation was carried out immediately when the temperature in a hot oven became $135^{\circ} \mathrm{C}$. In contrast, the draw ratio decreased with increasing annealing time and this tendency became considerable when the annealing time was beyond $15 \mathrm{~min}$.

\section{CONCLUSION}

From the above consideration, two main conclusions can be derived.

First, the crystal lamellae of dry gel films prepared from solution with the critical concentration $c^{*}(0.4 \mathrm{~g} / 100 \mathrm{ml})$ were confirmed to show the intermediate morphological properties between crystal lamellae prepared from a solution above $c^{*}$ and those prepared from that below $c^{*}$ on the basis of SAXS intensity distribution. That is, the scattered maxima 
from the specimens crystallized from the solution with the critical concentration bacame indistinct in comparison with the maxima of the specimens crystallized from the solution above the critical concentration. In comparison with the specimens crystallized from the solution below the critical concentration, however, the inverse relationship was found for the profile of the scattering maxima. Furthermore, the long period is the middle value when the specimens were prepared at the critical concentration. This indicates that as for the specimens crystallized from the solution with the critical concentration, the number of entanglements is the middle value in comparison with the number for the specimens crystallized with the solutions above and below the critical concentrations. This was the suitable number of entanglements which gave the best condition for facile drawability.

Secondly, the profile of the SAXS intensity distribution was measured for the annealed specimens on the basis of the two parameters, annealing time and annealing temperature, in order to study the long period and the orientational disorder or/and thickness fluctuation of crystal lamellae more quantitatively than in the previous works. ${ }^{5-7}$ With increase of annealing time (or annealing temperature), the SAXS intensity distribution becomes indistinct and the long period increases. This is due to an increase in entanglements. This increase causes a significant effect to promote the degree of preferential orientation of crystallites and the mechanical properties, especially when the annealing temperature is above $130^{\circ} \mathrm{C}$.

Acknowledgement. We thank to Prof. Tsujii and Prof. Niwa of Nara Women's University for their valuable comments and suggestions on X-ray instrument in Nara Women's University.

\section{REFERENCES}

1. P. Smith and P. J. Lemstra, J. Mater. Sci., 15, 505 (1980).

2. P. Smith and P. J. Lemstra, J. Polym. Sci., Polym. Phys. Ed., 19, 877 (1981).

3. P. Smith, P. J. Lemstra, J. P. L. Pijpers, and A. M. Kiel, Colloid Polym. Sci., 259, 1070 (1981).

4. M. Matsuo, K. Inoue, and N. Abumiya, Sen- $i$ Gakkaishi, 40, 275 (1984); M. Matsuo, K. Inoue, and N. Abumiya, Polym. Prepr. Jpn., 32, 841 (1983).

5. M. Matsuo and R. St. J. Manley, Macromolecules, 15, 985 (1982).

6. M. Matsuo and R. St. J. Manley, Macromolecules, 16, 1500 (1983).

7. M. Matsuo, M. Tsuji, and R. St. J. Manley, Macromolecules, 16, 1505 (1983).

8. D. A. Blackadder and J. S. Keniry, Macromol. Chem., 141, 211 (1971).

9. S. K. Bhateja, E. H. Andrews, and R. J. Young, $J$. Polym. Sci., 21, 523 (1983).

10. T. Oda, S. Nomura, and H. Kawai, J. Polym. Sci., A, 3, 1993 (1965).

11. S. Nomura, A. Asanuma, S. Suehiro, and H. Kawai, J. Polym. Sci., A-2, 9, 1991 (1971).

12. S. Nomura, M. Matsuo, and H. Kawai, J. Polym. Sci., Polym. Phys. Ed., 10, 2489 (1972).

13. C. Sawatari and M. Matsuo, Colloid Polym. Sci., in press. 